\title{
LES FONDEMENTS DE LA REVISION DE LA CONSTITUTION CONGOLAISE DU 18 FEVRIER 2006
}

\author{
Par BALINGENE KAHOMBO*
}

Résumé

Cet article examine les fondements juridiques et politiques qui caractérisent les initiatives de révision de la Constitution congolaise du 18 février 2006. Le constat de base indique que ce texte constitutionnel peine à résister à l'affirmation du pouvoir politique, qui voudrait, à tout prix, conformer les règles juridiques aux pratiques politiciennes. Il s'en suit le détournement du pouvoir constituant dérivé. Les conséquences d'un tel détournement sont nombreuses et dénotent quels en sont les visés réels. Leur dénominateur commun est constitué de la personnalisation du pouvoir et du renforcement du rôle du Chef de l'Etat, conduisant à une sorte d'hypertrophie de la fonction présidentielle, à travers notamment la présidentialisation du pouvoir constituant dérivé, l'extension et le renforcement des pouvoirs personnels du Chef de l'Etat, les tentatives d'empêcher l'alternance démocratique au pouvoir et l'anéantissement de l'indépendance du pouvoir judiciaire. L'article explore quelques pistes de solutions juridiques pour la protection de l'Etat de droit, en mettent en avant notamment l'élargissement de la composition du constituant dérivé aux assemblées provinciales et l'instauration d'un mécanisme de contrôle obligatoire de constitutionnalité de toute loi de révision constitutionnelle.

\section{Introduction}

En l'espace de moins de deux législatures (2006-2014), la Constitution du 18 février 2006 a déjà fait l'objet d'au moins trois initiatives de modification. En 2007, il y avait eu la proposition Tshibangu Kalala, ${ }^{1}$ qui n'avait été écartée, avant même son examen, que du fait de la volonté personnelle du Chef de l'Etat, depuis son discours du 6 décembre 2007 sur l'état de la nation. ${ }^{2}$ Toutefois, cette tentative d'amendement constitutionnel a été suivie par l'adop-

* Doctorant en droit à l'Université libre de Berlin, Diplômé d'études supérieures en droit public (Université de Kinshasa); chef de travaux à l'Université de Goma; chercheur à l'Institut pour la démocratie, la gouvernance, la paix et le développement en Afrique (IGDPA); membre du réseau international de recherche, Rule of Law Program for Sub-Saharan Africa, piloté par la Fondation Konrad Adenauer; magistrat/Juge (RDC). E-mail: internationaliste82@yahoo.fr.

1 Voir S. BOLLE, « RD Congo. Faut-il déjà réviser la Constitution de 2006 », http://www.la-constitut ion-en-afrique.org/, 27 novembre 2007.

2 Voir KABILA (J.), L'état de la nation: 2007-2008-2009, Kinshasa, Editions de la presse présidentielle, 2010, p.47. 
tion tout expresse, ${ }^{3}$ par le Parlement réuni en Congrès, de la loi de révision constitutionnelle du 20 janvier 2011, ${ }^{4}$ modifiant l'œuvre du constituant originaire en ses articles 71, 110, 126, 149, 197, 198, 218 et 226. Peu après, en 2013, un autre débat public a fait surface autour de la modification du nombre et de la durée des mandats présidentiels, à la suite de la publication de l'ouvrage tentaculaire d'Evariste Boshab, ${ }^{5}$ plaidant en faveur d'une nouvelle révision de la Constitution. ${ }^{6}$

Par conséquent, aussi jeune qu'elle soit, la Constitution du 18 février 2006 peine à résister à l'affirmation du pouvoir politique et à l'instabilité constitutionnelle qui en résulte. Les règles juridiques auraient vocation à s'adapter aux pratiques politiques, voire politiciennes, au lieu que la politique se soumette à la constitution ${ }^{7}$ et n'instaure la loi de la jungle ainsi que la culture de l'impunité. Mais, il n'y a rien de nouveau en cela. Il s'agit de la confirmation de l'histoire politique de la République démocratique du Congo (RDC), où l'instabilité constitutionnelle se caractérise, depuis 1960, par l'adoption d'un nombre très élevé des constitutions et la haute fréquence de leurs modifications. ${ }^{8}$ Aucun texte n'y a échappé, mais c'est la Constitution du 24 juin 1967 qui détient jusque-là le record de dixsept révisions constitutionnelles. ${ }^{9}$

Ceci montre à quel point le pays vacille entre l'affirmation constitutionnelle de l'Etat de droit et des détours fréquents, sinon des reculs permanents, vers la confiscation et l'exercice dictatorial du pouvoir d'Etat. La vérité est que l'instabilité constitutionnelle tant décriée est liée à la personnalisation du pouvoir et surtout au renforcement du rôle du Chef de l'Etat, tout puissant et à l'abri de tout contrôle. ${ }^{10}$

3 S. BOLLE, « Révision express », http://www.la-constitution-en-afrique.org/, $1^{\text {er }}$ mars 2011.

4 Loi n $11 / 002$ du 20 janvier 2011 portant révision de certains articles de la Constitution de la République démocratique du Congo du 18 février 2006.

5 Professeur de droit public et Secrétaire général du parti présidentiel, le PPRD (Parti du peuple pour la reconstruction et le développement).

6 E. BOSHAB, Entre la révision de la constitution et l'inanition de la nation, Bruxelles, Larcier, 2013, pp.127-295. Depuis lors, le champ du débat juridique s'est notamment étendu à la modification des modes de scrutin à l'élection du Président de la République et des députés provinciaux. A cet égard, notons que c'est la Commission électorale nationale indépendante (CENI) qui a visiblement rallumé les discussions et controverses suite à la présentation, le 30 janvier 2014, à l'Assemblée nationale, de sa feuille de route, intitulée « la RDC, deux ans après les élections de 2011. Etats des lieux et perceptives ", dans laquelle elle a suggéré l'élection des députés provinciaux au scrutin indirect, sachant que cette option conduirait inéluctablement à la révision de la Constitution. Cette suggestion a été entérinée par le gouvernement au mois de juin 2014.

7 A. MAMPUYA KANUNK'a TSHIABO, «Préface », in J.-L. ESAMBO KANGASHE, Le droit constitutionnel, Louvain-la-Neuve, Academia-L'Harmattan, 2013, p.13.

8 M. WETSH'OKONDA KOSO SENGA, Les textes constitutionnels congolais annotés, Kinshasa, Editions de la Campagne pour les Droits de l'Homme au Congo (CDHC-ASBL), 2010, p.7.

9 Ibid.

10 M. YABILI, Etat de droit: les contrôles de constitutionnalité par la Cour constitutionnelle, les cours et les tribunaux, Lubumbashi, PUL, 2012, p.23. Cet auteur donne sa lecture de la présidentialisation du régime en ces termes: «Le Chef de l'État a des fonctions constitutionnelles déjà fort 
Ce renforcement n'est pas une caractéristique d'un régime de type présidentiel. Il correspond plutôt aux pouvoirs absolutistes qui avaient été abolis par le principe séculaire de la séparation des pouvoirs et constitue, par conséquent, un recul dans le cheminement de l'Etat de droit. En RDC, cette prééminence politique et fonctionnelle accordée au Chef de l'Etat remonte à la Constitution de Luluabourg du $1^{\text {er }}$ août $1964 .{ }^{11}$ Mais, il semble qu'il s'agit, par ailleurs, d'une tendance constitutionnelle dans la majorité des Etats en Afrique sub-saharienne, ${ }^{12}$ conduisant à une sorte d'hypertrophie de la fonction présidentielle. ${ }^{13}$

Les nouvelles tentatives de modifier la Constitution du 18 février 2006 et de l'instrumentaliser menacent, ainsi, de rééditer un passé sombre, à la faveur de la dérive d'une majorité parlementaire mécanique, ${ }^{14}$ sans aucun égard à la préservation des acquis de l'Etat de droit et du jeu démocratique. Pourtant, l'État de droit est la norme de référence pour l'exercice du pouvoir, au point d'être proclamé trois fois par la Constitution elle-même : d'abord, dans l'exposé des motifs, où il est énoncé «... les préoccupations majeures... (d') instaurer

importantes, mais bien distinctes du pouvoir exécutif qui revient au gouvernement. Le principe est que le Chef de l'État ne prend pas de décisions, et par conséquent, il ne peut pas être interpellé, par absence d'objet et de responsabilité. Par contre, le gouvernement décide et agit, et de ce fait, il doit en répondre devant le peuple représenté par ses élus : les parlementaires (députés et sénateurs). Néanmoins, on a assisté à une présidentialisation du pouvoir, avec un Chef d'État qui se substitue au gouvernement pour décider et gérer directement, en particulier, les finances publiques. Pourtant, la Constitution n'autorise pas d'empiétement ; le Chef de l'État n'a pas de domaine réservé ; sa concertation avec le Premier ministre ne lui accorde pas le dernier mot, et, à titre de preuve, le Chef de l'État ne peut pas démettre le chef du gouvernement. L'investiture et la révocation du Premier ministre sont actionnées par le parlement, et la majorité des députés. On se trouve davantage dans un régime semi-parlementaire plutôt que semi-présidentiel. D'ailleurs, le Chef de l'État ne bénéficie pas d'une réelle immunité pénale, en cours d'exercice de ses fonctions (les articles 164, 166 et 167 de la Constitution indiquent que les délits spéciaux et infractions de droit commun commises « dans l'exercice des fonctions » restent poursuivables sur autorisation du Congrès) ».

11 Voir BALINGENE KAHOMBO, « L'expérience congolaise de l'Etat fédéral : la Constitution de Luluabourg revisitée ", http://www.la-constitution-en-afrique.org/, 24 mai 2010. On pourrait même remonter plus loin dans 1'histoire, quand on prend en considération les manœuvres politiques du Président Joseph Kasa Vubu en 1960. Il avait réussi à transformer le parlementarisme dualiste consacré par la Loi fondamentale du 19 mai 1960 en un parlementarisme moniste de type orléaniste. Il s'est ainsi permis de présenter un programme de gouvernement alors qu'il devait se contenter de régner sans gouverner. Il s'est, par la suite, arrogé le pouvoir de révoquer, de manière unilatérale, le Premier ministre, et de nommer son remplaçant qui n'était pas issu de la majorité parlementaire. L'idée était, à l'époque, qu'un Chef de l'Etat fort aurait permis de renforcer l'autorité publique et de préserver l'unité du pays, déjà déchiré par des divisions tribales, régionales et sécessionnistes. En tout état de cause, l'histoire renseigne que tous ces maux n'ont eu cesse de gangrener le pays, pourtant sous l'emprise des chefs d'Etat successifs détenteurs véritables de tous les pouvoirs d'Etat.

12 A. MAMPUYA KANUNK'a TSHIABO, op.cit., pp.9-10.

13 Ibid.

14 BALINGENE KAHOMBO, « L'originalité de la Cour constitutionnelle congolaise: son organisation et sa compétence », Librairie africaine d'études juridiques, vol.6, août 2011, p.15. 
un État de droit $»^{15}$; ensuite, le préambule de la Constitution proclame : «...Nous, Peuple congolais, animé par notre volonté commune de bâtir, au cœur de l'Afrique, un État de droit $»^{16}$; enfin, de façon plus radicale, le premier article de la Constitution proclame que «...la République démocratique du Congo est un État de droit $»{ }^{17}$

Dès lors, comment convient-il d'arrêter le pouvoir? Comment, juridiquement, est-il possible de combattre cette émergente instabilité constitutionnelle anti-démocratique, voire non-juridique, si on se réfère aux trois proclamations de l'Etat de droit, laquelle refait surface dans le pays? Quels mécanismes convient-il de mettre en place, à travers un réaménagement technique de la Constitution, pour, d'une part, contrecarrer le détournement persistant du pouvoir constituant dérivé et, d'autre part, renforcer et améliorer l'Etat de droit?

Cette étude met au cœur de l'analyse le danger du détournement du pouvoir constituant dérivé de la Constitution congolaise du 18 février 2006. Aussi tente-t-elle de démontrer, tour à tour, sa présidentialisation actuelle très prononcée (1), l'extension des pouvoirs personnels du Chef de l'Etat (2), les tentatives de remise en question du principe de l'alternance démocratique au pouvoir (3) et l'anéantissement de l'indépendance du pouvoir judiciaire (4), avant d'explorer, en définitive, quelques pistes de solutions juridiques pour contrecarrer la persistance de ce danger et protéger l'Etat de droit (5).

\section{La présidentialisation du pouvoir constituant dérivé dans la Constitution du 18 février 2006}

Par définition, le pouvoir constituant dérivé c'est celui que la procédure de révision constitutionnelle met en place. ${ }^{18}$ On l'appelle aussi pouvoir ou compétence de révision de la constitution. ${ }^{19}$ En RDC, il est consacré à l'article 218 de la Constitution du 28 février 2006. Ce pouvoir constituant est soit le peuple, soit le Parlement réuni en Congrès. Il s'agit de deux composantes nationales: l'une est sociale, l'autre est politique et institutionnelle. Le peuple est le souverain primaire; c'est lui qui a adopté la Constitution en cours. Aussi, a-t-il la compétence de se prononcer sur toute révision constitutionnelle, quelle qu'en soit l'initiative, en s'exprimant par référendum. ${ }^{20}$ Toutefois, le peuple n'est pas consulté, si le Parlement, réuni en Congrès (Assemblée nationale et Sénat), approuve une révision constitutionnelle à la majorité qualifiée de $3 / 5$ de ses membres. ${ }^{21}$

Par ailleurs, les deux pouvoirs constituants dérivés n'agissent pas proprio motu. Il faut qu'ils aient été mis en mouvement. En la matière, on distingue quatre types d'initiatives

15 Voir le point 3 de l'exposé des motifs.

16 Paragraphe 3.

17 Pour plus de détails, voir M. YABILI, op.cit., pp.97 et s.

18 V. CONSTANTINESCO et S. PIERRE-CAPS, Droit constitutionnel, Paris, PUF, 2004, p.191.

19 Idem, p.192.

20 Article 218, alinéa 3.

21 Idem, alinéa 4. 
concurrentes de révision constitutionnelle : 1) celle émanant du Président de la République ; 2) celle présentée par le gouvernement ; 2) celle portée par, distinctement, la moitié des membres de l'Assemblée nationale ou du Sénat ; 3) et celle émanant d'une fraction du peuple congolais, s'exprimant sous la forme d'une pétition signée par au moins 100.000 personnes et adressée à l'une de deux chambres du Congrès.

Dès lors, une première procédure voudrait que l'Assemblée nationale et le Sénat se prononcent directement sur le projet, la proposition ou la pétition portant révision de la Constitution. Siégeant séparément, l'une et l'autre en discute l'opportunité et la recevabilité ; puis, s'ils ne le rejettent pas, chacun l'adopte à la majorité absolue des chambres. C'est alors seulement que peut intervenir le vote décisif permettant au Congrès (Assemblée nationale et Sénat réunis ensemble) de s'en passer de la consultation directe du peuple, s'il approuve l'amendement constitutionnel à la majorité pré-qualifiée de $3 / 5$.

La seconde procédure se conçoit comme la conséquence de l'échec du vote du Congrès à pouvoir s'en passer de la consultation directe du peuple. Celui-ci devrait alors être convoqué au référendum. Mais, qui détient la compétence de l'y convoquer?

En effet, l'ancien alinéa 3 de l'article 218 de la Constitution était, sans aucune autre précision, libellé comme suit : «la révision n'est définitive que si le projet, la proposition ou la pétition est approuvée par référendum ». ${ }^{22}$ Mais, la loi constitutionnelle du 20 janvier 2011 l'a modifié en ajoutant au bout de cette disposition que la révision constitutionnelle est approuvée par référendum « sur convocation du Président de la République ». ${ }^{23} \mathrm{Il}$ s'agit ici d'une augmentation des prérogatives constitutionnelles du Chef de l'Etat. Pourtant, l'article 211 de la même Constitution aurait permis de penser que cette compétence était déjà attribuée à la Commission électorale nationale indépendante (CENI), dans la mesure où il lui confère, bien qu'en termes généraux, le pouvoir d'organiser « tout référendum », ${ }^{24} \mathrm{y}$ compris donc constitutionnel : non seulement son organisation matérielle, mais aussi, et avant tout, sa convocation. En d'autres termes, bien que le Président de la République ait acquis le droit d'initier une révision constitutionnelle, au moins était-il dépourvu du pouvoir de convoquer le peuple au référendum pour approuver son initiative ou de celle d'une mouvance politique qui serait à sa solde. Cette compétence appartenait à la CENI, de la même façon qu'elle a le pouvoir de convoquer le peuple aux différentes échéances électorales. ${ }^{25} \mathrm{Il}$ y avait donc là une importante limitation des pouvoirs du Chef de l'Etat, bien spécifique à la RDC, l'un des objectifs du constituant originaire étant de « contrer toute tentative de dérive dictatoriale $» .{ }^{26}$

Le soulignement est nôtre.

23 Idem.

24 Article 211, alinéa 2.

25 Voir, par exemple, l'article 73 de la Constitution s'agissant de l'élection du Président de la République. Cet article dispose: «Le scrutin pour l'élection du Président de la République est convoqué par la Commission électorale nationale indépendante, quatre-vingt dix jours avant l'expiration du mandat du Président en exercice».

26 Voir le point 3 de l'exposé des motifs de la Constitution. 
Il faut aussi relever que le constituant dérivé n'a fourni aucune explication l'ayant motivé à attribuer au Président de la République ce nouveau pouvoir constitutionnel. Mais, on peut avancer deux principales hypothèses. D'abord, il peut arriver que le Chef de l'Etat n'ait pas le contrôle du Congrès, en particulier en période de cohabitation, ou qu'il ait des difficultés à convaincre les parlementaires (congressistes) à pouvoir approuver son initiative $\mathrm{d}$ 'amendement constitutionnel à la majorité qualifiée qu'exige la Constitution. Ensuite, la CENI étant en principe indépendante, il n'est pas sûr qu'elle puisse convoquer tel ou tel autre référendum constitutionnel, même sur demande présidentielle expresse. Face à ces inquiétudes de nature politicienne, la solution n'était plus que celle d'accorder au Président de la République lui-même le pouvoir de convoquer le peuple au référendum, avec tout le risque de tripatouillage du vote comme c'est souvent le cas en Afrique. ${ }^{27}$ Au surplus, il s'agit d'une compétence discrétionnaire : seul le Chef de l'Etat décide de l'opportunité de convoquer le référendum constitutionnel, tandis que la CENI est tenue, en simple exécutant de la volonté présidentielle, de procéder à son organisation matérielle. Cette confusion des pouvoirs conduit à conclure à la présidentialisation du constituant dérivé de la Constitution du 18 février 2006.

En revanche, en période de concordance entre l'exécutif et la majorité parlementaire, ce nouveau pouvoir présidentiel apparaît plutôt comme une précaution politique en faveur du Chef de l'Etat. En pratique, la majorité qualifiée du Congrès qu'exige la Constitution pour sa propre modification ne serait pas difficile à atteindre, si bien que la consultation du souverain primaire devient presque aléatoire. L'argent corrompt la politique ${ }^{28}$ et la majorité au pouvoir obéit au mot d'ordre des chefs des partis ou « autorités morales ", ${ }^{29}$ selon une expression désormais célèbre en RDC.

D'où fut adoptée la loi $n^{\circ} 11 / 002$ du 20 janvier 2011 portant révision de certains articles de la Constitution de la République démocratique du Congo du 18 février 2006. Cette initiative trouve ses racines profondes dans les travaux de la Commission interinstitutionnelle qui fut constituée en 2010 pour faire l'évaluation de l'application de l'œuvre du constituant originaire, à peine vieille de cinq ans. Cependant, sa proposition ne fut officiellement invoquée que le 3 janvier 2011. La procédure devant le Congrès pour l'adoption de la modification constitutionnelle ne prit que cinq jours, soit un temps record plus court que

27 Voir K. AFO ABI, La transparence des élections en droit public africain. A partir des cas béninois, sénégalais et togolais, thèse de doctorat en droit, cotutelle, Université de Lomé et Université Montesquieu-Bordeaux IV, 2013, $538 \mathrm{p}$.

28 Voir P. PUNGA, RD Congo entre démocratie et corruption politique : sortir de l'ornière pour une gouvernance démocratique, communication présentée à la $13^{\text {ème }}$ Assemblée générale du Conseil pour le développement de la recherche en sciences sociales en Afrique (CODESRIA), Rabat (Maroc), du 5 au 9 décembre 2011.

29 A. MBATA, La diaspora congolaise en Afrique du Sud recommande un dialogue véritablement national et inclusif, rejette les concertations nationales, désapprouve les prétendus délégués de la diaspora et lance un vibrant appel à tous les congolais de l'étranger, commentaires de la déclaration du 10 août 2013 de la diaspora congolaise établie en Afrique du Sud contre la révision de l'article 220 de la Constitution, 2013, p.3. 
celui que prend normalement le vote des lois organiques et ordinaires. Cette rapidité n'est pas justifiable par le fait que la révision constitutionnelle a été adoptée en session extraordinaire du Parlement et en procédure d'urgence : les questions de forme et de fond devaient suffisamment faire l'objet d'un véritable débat démocratique et citoyen. Mais, ce fut tout simplement de la tricherie politique, ${ }^{30}$ tel que le témoigna le vote monolithique des parlementaires nationaux.

Ainsi, « le 11 janvier, l'Assemblée nationale, à l'issue d'une plénière houleuse, approuve la proposition de révision, par 324 voix pour, 2 contre et 1 abstention. Le vote a eu lieu après le départ de l'opposition, en l'absence de 173 des 500 députés. Le 13 janvier, le Sénat approuve à son tour la proposition de révision, par 71 voix pour, 1 contre et 9 abstentions. 27 sénateurs n'ont pas pris part au vote. L'Assemblée nationale et le Sénat, réunis en Congrès du 14 au 15 janvier, adoptent la loi de révision, par 485 voix pour, 8 contre et 11 abstentions. La majorité des $3 / 5$ des 608 parlementaires a été largement atteinte ». ${ }^{31}$

Toujours est-il que le pouvoir constituant dérivé a adopté une révision constitutionnelle cavalière à des fins personnelles, étrangères à l'amélioration de l'Etat de droit et de la démocratie. Le Chef de l'Etat en a été le principal bénéficiaire.

\section{L'extension et le renforcement constitutionnels des pouvoirs personnels du Chef de l'Etat}

La loi de révision constitutionnelle du 20 janvier 2011, dont l'article $1^{\mathrm{er}}$ modifie les articles 197, 198 et 218 de la Constitution du 18 février 2006, augmente les prérogatives constitutionnelles du Chef de l'Etat, alors que celles-ci étaient déjà très importantes : commandant suprême des forces armées et président du Conseil supérieur de la défense (article 83) ; nomination du Premier ministre et des autres membres du gouvernement (article 78); investiture des gouverneurs et vice-gouverneurs de province (article 80); Président de la Conférence des gouverneurs de provinces (article 200); nomination de tous les magistrats (article 82 ) ; nomination à d'autres principales fonctions dans l'Etat : ambassadeurs, officiers généraux et supérieurs de l'armée et de la police, mandataires des entreprises publiques, hauts fonctionnaires de l'administration publique, etc. (article 81); déclaration de guerre (articles 86 et 143), de l'état d'urgence ou de siège (articles 85, 144 et 145), etc.

Depuis 2011, de nouvelles prérogatives présidentielles sont ajoutées. Il y a la convocation du référendum constitutionnel (article 218). En matière de décentralisation, l'article 197, alinéa 7, lui confère le pouvoir de dissoudre une assemblée provinciale, et l'article 198, alinéa 10, celui de révoquer un gouverneur de province.

Cette tendance à vouloir toujours augmenter ou renforcer les pouvoirs du Chef de l'Etat avait déjà été sévèrement critiquée lors du dépôt, le 5 novembre 2007, de la proposition de

30 A. MAMPUYA KANUNK’a TSHIABO, « La panique, le mensonge et la tricherie ", http:// www.lephareonline.net/lephare/index.php?option $=$ com_content\&view=article\&id=3083:lapanique-le-mensonge-et-la-tricherie\&catid=44:rokstories\&Itemid=106, 6 janvier 2011.

31 BOLLE (St.), « Révision express », op.cit. 
révision constitutionnelle à l'initiative du député Tshibangu Kalala. ${ }^{32}$ Bien que soutenue par 310 députés de la mouvance présidentielle, cette proposition échoua ${ }^{33}$ : en plus du tolet général qu'elle avait provoqué, elle était rejetée par le Président de la République lui-même, s'exprimant là-dessus dans son discours sur l'état de la nation en décembre 2007 en ces termes:

Je ne peux en finir avec les réformes juridiques sans nous mettre tous en garde contre la tentation de vouloir régler tout dysfonctionnement éventuel des institutions par une révision constitutionnelle. En principe, la loi fondamentale d'un pays ne devrait être modifiée qu'en cas d'extrême nécessité, et uniquement dans l'intérêt supérieur de la Nation. ${ }^{34}$

Depuis lors, l'extension des fonctions du Chef de l'Etat a envahi le domaine législatif. On lui en attribue toujours davantage dans un environnement qui provoque moins de controverses et de tensions politiques, puisque l'opinion semble plus intéressée par l'intégrité du texte constitutionnel que par celle des lois de la République.

1. L'exemple de la loi du 31 juillet 2008 relative à la libre administration des provinces

Dans le domaine de la décentralisation, la loi du 31 juillet 2008 sur la libre administration des provinces a confié au Président de la République le pouvoir de prendre acte, par simple ordonnance contresignée par le Premier ministre, ${ }^{35}$ de la dissolution de plein droit d'une assemblée provinciale, telle que constatée par le Président du Sénat et celui de l'Assemblée nationale. ${ }^{36}$ En outre, cette loi a autorisé l'éviction d'un gouverneur de province par son assemblée provinciale, à l'initiative du pouvoir central, y compris le Chef de l'Etat. L'astuce juridique en la matière a consisté à introduire dans la loi la notion de dédoublement fonctionnel qui fait du gouverneur à la fois chef de l'exécutif provincial et représentant du gouvernement central en province pour finalement permettre son contrôle direct par le pouvoir central. ${ }^{37}$

Ce sont ces prérogatives présidentielles qui ont visiblement été renforcées par la révision constitutionnelle du 20 janvier 2011 dans le libellé nouveau des articles 197, alinéas 7 et 8, et 198, alinéa 10, de la Constitution. L'article 197 ainsi révisé attribue au chef de l'Etat la compétence, non plus de prendre acte, mais de dissoudre carrément une assemblée provinciale, tandis que le nouvel alinéa 10 de l'article 198 lui donne le pouvoir de révoquer un gouverneur de province.

32 A. MAMPUYA KANUNK'a TSHIABO, La Constitution : la révision n'est pas une urgence, entretien avec le journal le Phare, rapporté par S. BOLLE, « RD Congo. Faut-il déjà réviser la Constitution de 2006 », http://www.la-constitution-en-afrique.org/, 27 novembre 2007.

33 Voir M. WETSH'OKONDA KOSO et V. MAKIDI KOMBE, «L'échec de l'initiative de révision constitutionnelle du 5 novembre 2007 », http://www.la-constitution-en-afrique.org, 17 juillet 2008.

34 J. KABILA, L'état de la nation...op.cit., p.47.

35 Article 20, alinéa 2.

36 Article 20, alinéa 1.

37 Lecture combinée des articles 63, 66 et 67 de la loi sur la libre administration des provinces. 
Deux arguments majeurs ont conduit à cette extension des prérogatives présidentielles sur les provinces. Primo, le statut de dédoublement fonctionnel du gouverneur de province, qui est un emprunt de la Constitution de Luluabourg du $1{ }^{\text {er }}$ août $1964 .{ }^{38}$ On n'a plus voulu garder une option visiblement compatible avec la volonté du premier constituant du pays, en exhumant l'institution de Commissaire d'Etat que la Loi fondamentale du 19 mai 1960 relative aux structures du Congo ${ }^{39}$ chargeait de représenter le pouvoir central en province, sans toucher au gouverneur qui était l'un des rouages essentiels de la traduction politique et juridique de l'autonomie de chaque province. Mais, en 1964, le choix du dédoublement fonctionnel du gouverneur de province fut justifié par la Commission constitutionnelle de Luluabourg pour éviter des frictions qu'il pouvait y avoir entre le chef de l'exécutif provincial et le commissaire d'Etat. ${ }^{40} \mathrm{Il}$ semble que c'est cet argument historique qui a influencé le législateur congolais de $2008 .{ }^{41} \mathrm{Il}$ palliait, ainsi, au silence du constituant originaire, alors qu'en droit public, ce qui n'est pas interdit n'est pas permis non plus, car les compétences sont toujours d'attribution.

Secundo, le constituant dérivé a voulu résoudre le problème posé par le diagnostic d'un 《 fonctionnement laborieux des institutions provinciales ${ }^{42}$ dans la majorité des provinces, à cause des crises répétées dues au recours aux motions de censure ou de défiance par les assemblées provinciales. Toutefois, des crises sont nées en provinces parce que le juge constitutionnel a précisément été politiquement instrumentalisé et incité à empêcher le contrôle normal des gouverneurs et des exécutifs provinciaux par leurs assemblées provinciales, alors que l'usage, même répété, des motions de défiance ou de censure ne saurait,

38 Article 103. Voir BALINGENE KAHOMBO, « L'expérience congolaise de l'Etat fédéral : la Constitution de Luluabourg revisitée », op.cit.

39 Articles 180 à 184.

40 Mémoire explicatif de la Constitution, présenté au Gouvernement de la République par le Secrétariat de la Commission constitutionnelle gouvernementale, Moniteur Congolais, $6^{\text {ème }}$ année, numéro spécial, 5 octobre 1965, p.95.

41 P. PUNGA KUMAKINGA, « Problématique de la conformité à la Constitution de la loi organique sur les entités territoriales décentralisées en République démocratique du Congo. Regard sur la Commune de Mont-Ngafula dans la ville de Kinshasa », Librairie africaine d'études juridiques, vol.6, août 2011, p.102.

42 Voir Exposé des motifs de la proposition de loi portant révision de la Constitution de la République démocratique du Congo du 18 février 2006, point 5. 
logiquement, être qualifié de dysfonctionnement. ${ }^{43}$ Cela fait partie du travail normal de contrôle des assemblées provinciales.

La vérité est que cette extension des compétences du Chef de l'Etat est en contradiction avec la volonté du constituant originaire de 2006. De ce fait, la révision était elle-même contraire à la Constitution. L'inconstitutionnalité tient ici au fait que, originairement, on a instauré une nette séparation entre les deux principaux échelons du pouvoir d'Etat : la province régionalisée et le pouvoir central. Chaque échelon dispose d'organes propres et autonomes, et jouit d'une légitimité constitutionnelle distincte et séparée. C'est ainsi que l'article 195 indique que « les institutions provinciales sont: 1.1'Assemblée provinciale; 2.le Gouvernement provincial ». L'assemblée provinciale est composée des députés, élus au suffrage universel direct, tandis que le gouverneur, chef du gouvernement provincial, est désigné par elle au scrutin indirect et lui est politiquement redevable de la gestion de la province. L'autonomie politique provinciale est ainsi bien déterminée par rapport au pouvoir central qui comprend le Président de la République, le Parlement (Sénat et Assemblée nationale) et le Gouvernement central. ${ }^{44}$ Par conséquent, cette légitimité propre et séparée implique l'interdiction de nomination et révocation des membres des institutions politiques provinciales, d'une part, et, d'autre part, fait obstacle à la dissolution de celles-ci par le pouvoir central. Raisonner autrement contreviendrait à l'article 220 de la Constitution qui interdit toute modification constitutionnelle réduisant l'autonomie des provinces. ${ }^{45}$ A plus forte raison, la loi du 31 juillet 2008 sur la libre administration des provinces est anticonstitutionnelle, dans la mesure où elle a placé celles-ci sous une sorte de tutelle déguisée du pouvoir central. ${ }^{46}$

43 A. MAMPUYA KANUNK'A-TSHIABO, « Au feu! L'article 220 violé », Le Potentiel, Kinshasa, 6 janvier 2011. Voir http://www.lepotentiel.com/afficher_article.php?id_edition=\&id_article=1055 07, consulté le 1er mars 2011. Cet auteur écrit : « La difficulté est apparue dès le moment où, ignorant le droit, la Cour suprême de Justice s'est crue juridiquement fondée à mettre à bas une motion votée par une assemblée et à anéantir ainsi une prérogative constitutionnelle de l'assemblée parlementaire, ce qui est une ingérence inadmissible non seulement dans le fonctionnement mais aussi dans les attributions constitutionnelles d'une autre institution; cela pourrait inspirer un Premier ministre frappé par une motion de censure pour refuser sa sanction et saisir la Cour ou un président de l'assemblée provinciale de solliciter le secours d'un organe judiciaire, dénaturant complètement l'esprit du régime du contrôle parlementaire. C'est cette ignorance du droit par les juges de la Cour qui a introduit le désordre et non l'exercice de leur prérogative constitutionnelle par les assemblées provinciales.

44 De notre point de vue, le pouvoir judiciaire, qui est en principe indépendant du législatif et de l'exécutif, ne fait donc pas partie du pouvoir central, bien que celui-ci détienne la compétence constitutionnelle de déterminer son organisation et son fonctionnement. Il s'agit plutôt de l'arbitre entre les deux échelons du pouvoir d'Etat.

45 A. MAMPUYA KANUNK'A-TSHIABO, « Au feu! L'article 220 violé », op.cit.

46 BALINGENE KAHOMBO, «L'expérience congolaise de l'Etat régional. Le contrôle politique du pouvoir central sur les provinces : vers une tutelle déguisée », http://www.la-constitution-en-afriqu e.org/, 12 septembre 2013. 


\section{Le défaut de vigilance du juge constitutionnel}

Cette anomalie n'aurait pas dû échapper à la vigilance du juge constitutionnel. En effet, l'article 160 de la Constitution prescrit:

Les lois organiques, avant leur promulgation, et les Règlements intérieurs des Chambres parlementaires et du Congrès, de la Commission électorale nationale indépendante ainsi que du Conseil supérieur de l'audiovisuel et de la communication, avant leur mise en application, doivent être soumis à la Cour constitutionnelle qui se prononce sur leur conformité à la Constitution. Aux mêmes fins d'examen de la constitutionnalité, les lois peuvent être déférées à la Cour constitutionnelle, avant leur promulgation, par le Président de la République, le Premier ministre, le Président de l'Assemblée nationale, le Président du Sénat ou le dixième des députés ou des sénateurs.

Par conséquent, il est curieux de constater que la Cour suprême de Justice (CSJ), juridiction constitutionnelle transitoire aux termes de l'article 223 de la Constitution, ait déclaré conforme à celle-ci la loi du 31 juillet 2008 relative à la libre administration des provinces. ${ }^{47}$ Pire, la CSJ s'est délibérément abstenue de se prononcer sur les nombreuses requêtes en contrôle de constitutionnalité du nouvel amendement constitutionnel dont elle a été saisi, ${ }^{48}$ en violation de l'article 160 in fine de la Constitution. ${ }^{49}$

Le Président de la République peut, désormais, faire ce qu'il veut. L'encadrement de ses nouveaux pouvoirs est insignifiant. Tout d'abord, pour révoquer un gouverneur de province ou dissoudre une assemblée provinciale, une seule condition de fond lui est imposée : l'existence d'une crise politique grave et persistante qui menace d'interrompre le fonctionnement régulier des institutions provinciales. Cependant, le constituant ne définit pas la consistance matérielle d'une telle crise. Visiblement, il appartient au Chef de l'Etat d'apprécier quand et comment cette condition est réalisée et de choisir librement l'option constitutionnelle qui lui semble appropriée. En outre, en la forme, il n'a l'obligation que de consulter les bureaux de l'Assemblée nationale et du Sénat, sans que les avis et considérations émanant d'eux puissent le lier. Néanmoins, l'ordonnance de dissolution d'une assemblée provinciale ou de révocation d'un gouverneur de province, bien que délibérée en Conseil des ministres, exige le contreseing du Premier ministre. En effet, cette ordonnance est prise dans le cadre de l'article 197 qui ne rentre pas dans les quatre cas exceptionnels de l'article 79 et où le Président de la République a le droit de signer seul. ${ }^{50}$

Cette hyper-présidentialisation de la régionalisation provinciale crée un climat politique malsain, car il pourrait pousser tous les membres des institutions politiques provinciales et surtout les gouverneurs de provinces à se mettre dans le giron du Président de la

47 P. PUNGA KUMAKINGA, « Problématique de la conformité à la Constitution de la loi organique sur les entités territoriales décentralisées en République démocratique du Congo... », op.cit., p.93.

48 C'est le cas notamment d'une requête déposée devant lui depuis le 9 mars 2011.

49 Cet article dispose : « La Cour constitutionnelle statue dans le délai de trente jours. Toutefois, à la demande du Gouvernement, s'il y a urgence, ce délai est ramené à huit jours ».

50 L'article 79 fait exception pour les articles 78, alinéa premier, 80, 84 et 143 de la Constitution. 
République pour espérer sauvegarder durablement leurs mandats : transformation en agents d'exécution de la volonté du Chef de l'Etat, approvisionnement en ressources, battre campagne pour sa réélection, ainsi qu'on l'a observé en novembre 2011, etc. Ceci compromet la démocratie à la base et viole virtuellement, mais de façon certaine, le droit de l'opposition à s'arroger le gouvernement d'une province où elle aurait gagné les élections. Cette présidentialisation s'accompagne du renvoi aux calendes des grecs de l'installation de 26 nouvelles provinces, ${ }^{51}$ dont pourtant le constituant originaire avait voulu la matérialisation dans un délai de 36 mois suivant la mise en place des institutions politiques de la troisième république. Désormais, l'article 226 révisé de la Constitution prévoit : « une loi de programmation détermine les modalités d'installation de nouvelles provinces citées à l'article 2 de la présente Constitution $»{ }^{52}$ C'est comme si on tentait d'empêcher l'implémentation des fondements mêmes de la Constitution.

En 2006, tous les acteurs politiques avaient remis leurs tabliers et méthodes de plusieurs années de dictatures et de conflits. Le souverain primaire consulté par voie de référendum a installé une " troisième république ». Outre la caractéristique de la triple déclaration d'un Etat de droit, la Constitution de 2006 met fin à la dictature et à toute entreprise de conquête et de conservation du pouvoir par la force, pour ne retenir que la seule voie démocratique des urnes. La majorité exerce le pouvoir avec une opposition reconnue. Dans ces circonstances, l'enjeu constitutionnel est d'assurer à tout moment que la majorité d'aujourd'hui ait, suivant le verdict populaire, l'occasion de devenir l'opposition de demain, et vice versa. Autant dire l'alternance démocratique au pouvoir, un principe fondamental du constituant de 2006.

\section{Les tentatives d'empêcher l'alternance démocratique au pouvoir}

Deux facteurs permettent d'avancer vers un blocage de l'alternance démocratique au pouvoir en RDC. Tout d'abord, la loi constitutionnelle du 20 janvier 2011 a modifié le mode de scrutin à l'élection présidentielle. L'article 71 modifié de la Constitution a alors supprimé l'élection du Chef de l'Etat au scrutin majoritaire à deux tours pour autoriser son élection à un seul tour à la majorité simple des suffrages exprimés. Ensuite, depuis 2013, les débats achoppent autour de la révision du nombre et de la durée des mandats présidentiels. Cette éventuelle révision marque toute sa différence avec la modification du mode de scrutin à l'élection présidentielle par le fait qu'elle touche de plein fouet aux dispositions constitutionnelles intangibles, verrouillées par l'article 220 de la Constitution.

Il s'agit, dans les deux cas, de faciliter le maintien au pouvoir du Chef de l'Etat en fonction en bénéficiant de la majorité parlementaire. Ces deux projets de révision empiètent volontairement sur le principe de l'alternance démocratique au pouvoir.

51 Il s'agit des provinces ci-après : Kinshasa, Bas-Uélé, Equateur, Haut-Lomami, Haut-Katanga, Haut-Uélé, Ituri, Kasaï, Kasaï oriental, Kasaï central, Kwango, Kwilu, Lomami, Lwalaba, Mongala, Nord-Kivu, Nord-Ubangi, Sankuru, Sud-Kivu, Sud-Ubangi, Tanganyika, Tshopo et Tshuapa.

52 Alinéa 1. 
1. La modification cavalière du mode de scrutin à l'élection du Président de la République

L'adoption du nouvel article 71 de la Constitution est intervenue au cours de la préparation des élections du 28 novembre 2011, alors que le président Joseph Kabila préparait le renouvellement de son mandat de cinq ans, obtenu en 2006. Formellement, le constituant dérivé n'a pas clarifié les raisons profondes à la base de cette révision constitutionnelle. Il a simplement indiqué que « certaines dispositions [de la Constitution] se sont révélées handicapantes et inadaptées aux réalités politiques et socioéconomiques de la République démocratique du Congo $»,{ }^{53}$ sans toutefois préciser de quelle réalité il s'agit.

On sait, néanmoins, que le gouvernement a avancé deux arguments majeurs en faveur de cette réforme constitutionnelle.

Primo, il a pris appui sur l'expérience des élections présidentielles de 2006 qui, par l'effet de la bipolarisation au second tour entre Joseph Kabila et Jean-Pierre Bemba, ont divisé le pays en deux camps (Est-Ouest), semé un climat de tension entre les populations swahiliphones de l'Est et lingalaphones de l'Ouest, au point d'aboutir une véritable dérive identitaire.

Secundo, le gouvernement avait invoqué le coût exorbitant d'une élection à deux tours, parce que les finances de l'Etat étaient peu reluisantes. Cependant, aucun budget de dépenses électorales n'avait été avancé pour justifier non seulement leur importance, mais surtout leur impact qui aurait été négatif pour le pays ; et pour en arriver à une révision constitutionnelle, il fallait la preuve qu'il allait en être ainsi pour toutes les échéances électorales futures. Ces preuves financières n'avaient jamais été avancées ${ }^{54}$. En plus, le gouvernement omettait le fait que le cycle électoral envisagé n'était pas limité aux deux élections combinées (présidentielle et parlementaire) et que, de toute façon, le gouvernement allait dépenser pour organiser les élections à la députation provinciale, lesquelles avaient été combinées, en 2006, avec le second tour de l'élection présidentielle. D'ailleurs, l'argument financier paraissait un peu surprenant dès le moment où le gouvernement se vantait d'avoir atteint le point d'achèvement de l'initiative $\mathrm{PPTE}^{55}$ auprès des institutions financières internationales avec pour effet l'annulation de la dette extérieure du pays et le gain mensuel de 50 million de dollars américains pour le budget de l'Etat. Aussi, la fragilité de cet argument financier avait-elle conduit à son abandon pur et simple dans la proposition de loi de révi-

53 Exposé des motifs de la loi n¹1/002 du 20 janvier 2011 portant révision de certains articles de la Constitution de la République démocratique du Congo du 18 février 2006, paragraphe 2.

54 En 2013, dans son discours sur l'état de la nation, le Président Kabila avancera, à nouveau, des raisons financières pour proposer que l'élection des députés provinciaux se fasse au suffrage indirect. L'impact financier n'a pas été produit ni démontré. Toutefois, c'est cette proposition que la CENI a reprise, en janvier 2014, dans sa feuille de route, intitulée « la RDC, deux ans après les élections de 2011. Etats des lieux et perceptives », soumise à l'Assemblée nationale pour examen et décision.

55 Pays pauvres très endettés. 
sion constitutionnelle pour ne reprendre que des motifs d'ordre sécuritaire et de cohésion nationale. ${ }^{56}$

En réalité, la modification de l'article 71 de la Constitution ne pouvait rationnellement s'expliquer que par des calculs et stratégies électoraux. En effet, le président Joseph Kabila n'était plus sûr de remporter les élections. Premièrement, contrairement au scrutin de 2006, il courait le risque de perdre son électorat du Nord et Sud-Kivu, présumés acquis au leader de l'Union pour la nation congolaise (UNC), Vital Kamerhe, dissident du parti présidentiel, le PPRD ${ }^{57}$, et ancien président de l'Assemblée nationale, qui avait été forcé à démissionner en 2009 en raison de sa désapprobation des arrangements sécuritaires secrets entre la RDC, le Rwanda et la rébellion du $\mathrm{CNDP}^{58}$, futur groupe armé connu sous le nom du M23. Deuxièmement, on notait le retour en force du vieil et historique opposant, Etienne Tshisekedi, dont les partisans sont concentrés à Kinshasa, au Bas-Congo, au Kasaï occidental et oriental. Troisièmement, enfin, il ne pouvait pas compter sur l'électorat de l'Equateur, province d'origine de son challenger de 2006, Jean-Pierre Bemba, en détention auprès de la Cour pénale internationale (CPI). Dès lors, la seule chance de pouvoir l'emporter consistait dans la modification du mode de scrutin dans l'espoir d'entrer en compétition électorale face à des candidats multiples d'une opposition divisée, entraînant l'émiettement des voix à ses dépens, et empêchant, de toute façon, leur fédération/coalition lors d'un second tour finalement supprimé : le Président de la République étant désormais élu au suffrage universel direct à seul tour ${ }^{59}$. Cette révision constitutionnelle était donc taillée sur mesure. Par conséquent, elle relève d'une personnalisation conjoncturelle des règles de jeu démocratique au profit du Chef de l'Etat pour empêcher l'alternatince au pouvoir, au mépris de la volonté exprimée du constituant originaire en $2006 .^{60}$

Il faut aussi noter que ce type de révision cavalière des normes constitutionnelles, en l'absence de tout consensus politique, ${ }^{61}$ est interdit par le "Code panafricain de la démocra-

56 Exposé des motifs de la proposition de loi portant révision de la Constitution de la République démocratique du Congo du 18 février 2006, point 1.

57 Parti du peuple pour la reconstruction et la démocratie.

58 Congrès national pour la défense du peuple.

59 Pour plus de détails, voir BALINGENE KAHOMBO et M. WETSH'OKONDA KOSO, Le pari $d u$ respect de la vérité des urnes en Afrique. Analyse des élections présidentielles et législatives du 28 novembre 2011 en République démocratique du Congo, Bruxelles, éditions 11.11.11. Combattons l'injustice, 2014, pp.145-153.

60 Selon lui, l'une des préoccupations majeures qu'il a eue au moment de l'adoption de la Constitution du 18 février 2006 consistait à chercher comment "assurer l'alternance démocratique au pouvoir".

61 A cet égard, l'article 10, point 2, de la Charte africaine de la démocratie, des élections et de la gouvernance dispose : « Les Etats parties doivent s'assurer que le processus d'amendement ou de révision de leur Constitution repose sur un consensus comportant, le cas échéant, le recours au référendum ». 
tie, des élections et de la gouvernance", ${ }^{62}$ notamment par l'article 23 de la Charte africaine de la démocratie, des élections et de la gouvernance, ${ }^{63}$ qui qualifie de "changement anticonstitutionnel de gouvernement", crime international passible de sanctions par l'Union africaine (UA), « tout amendement ou toute révision des constitutions ou des instruments juridiques qui porte atteinte aux principes de l'alternance démocratique [au pouvoir] $»{ }^{64}$ Bien que la RDC n'ait pas encore ratifiée cette Charte, elle avait, au moins, l'obligation morale de ne pas attenter à son objet et à son but en tant que pays signataire. En outre, cette Charte ne lui était pas moins obligatoire dans la mesure où, pour pallier l'insuffisance de ses ratifications par les Etats africains, ses dispositions avaient déjà été rendues obligatoires, par le biais de la célèbre décision de $2010,{ }^{65}$ à l'égard de tous les Etats membres de l'UA et, par application directe et immédiate, ${ }^{66}$ pour tous les "citoyens africains". Au surplus, cet amendement constitutionnel contrevenait tout de même au Protocole sur la démocratie et la gouvernance, adopté dans le cadre de la CIRGL, ${ }^{67}$ dont la RDC est membre, puisqu'il interdit toute « réforme substantielle de la loi électorale dans les six (6) mois précédents les élections sans le consentement de la majorité des acteurs politiques et sociaux $\gg{ }^{68}$

A cette illicéité s'ajoute maintenant l'inconstitutionnalité d'une éventuelle modification du nombre et de la durée des mandats présidentiels, qui découle, de façon flagrante, du texte constitutionnel lui-même.

62 Nous entendons par là, en particulier, la Déclaration de l'OUA sur les principes régissant les élections démocratiques en Afrique, la Déclaration de Lomé sur la réaction de l'OUA en cas de changement anticonstitutionnel de gouvernement, l'Acte constitutif et son Protocole relatif à la création du Conseil de paix et de sécurité (CPS) de l'UA, le Mécanisme africain d'évaluation par les pairs (MAEP) et la Charte africaine de la démocratie, des élections et de la gouvernance.

63 Cette Charte a été adoptée par la Conférence de l'UA à Addis-Abeba (Ethiopie), le 30 janvier 2007. Elle est entrée en vigueur le 15 février 2012.

64 Point 5.

65 Décision Assembly/AU/Dec.269 (XIV) Rev. 1 sur la prévention des changements anticonstitutionnels de gouvernement et le renforcement de l'Union africaine à gérer de telles situations, adoptée par la Conférence de l'Union, lors de sa $14^{\text {ème }}$ session ordinaire tenue à Addis-Abeba (Ethiopie), du 31 janvier au 2 février 2010.

66 Ce caractère des décisions panafricaines a été réaffirmé dans la Décision Assembly/AU/Dec.109 (VI), adoptée par la Conférence de l'Union, lors de sa sixième session ordinaire, tenue à Khartoum (Soudan), du 23 au 24 janvier 2006. Lire aussi BALINGENE KAHOMBO et J. SEGIHOBE BIGIRA, «L'état de l'intégration juridique panafricaine : essai d'analyse du pouvoir réglementaire de l'Union africaine ", Annales de la Faculté de droit, Université de Kinshasa, 2013, pp.356-401.

67 Conférence internationale sur la région des grands lacs.

68 Article 4. La loi électorale doit être entendue ici au sens large, y compris les règles constitutionnelles applicables aux élections. 
2. La voie vers une éventuelle modification du nombre et de la durée des mandats présidentiels

L'article 70 de la Constitution prévoit que le Président de la République est élu pour un mandat de cinq ans une fois renouvelable, ${ }^{69}$ tandis que l'article 220 verrouille cette disposition en ces termes :

La forme républicaine de l'Etat, le principe du suffrage universel, la forme représentative du Gouvernement, le nombre et la durée des mandats du Président de la République, l'indépendance du pouvoir judiciaire, le pluralisme politique et syndical, ne peuvent faire l'objet d'aucune révision constitutionnelle. ${ }^{70}$

Est formellement interdite toute révision constitutionnelle ayant pour objet ou pour effet de réduire les droits et libertés de la personne, ou de réduire les prérogatives des provinces et des entités territoriales décentralisées.

Il s'agit là de ce qu'on qualifie des dispositions intangibles de la Constitution, mais qui font leur première apparition dans l'histoire constitutionnelle de la RDC. Evidemment, il ne s'agit pas des règles juridiques éternelles. Elles peuvent disparaître si ce texte constitutionnel est aboli par l'adoption d'une nouvelle constitution.

Il est aussi utile, avant toute chose, de situer la question dans son véritable contexte. L'idée même d'amender l'article 70 et de modifier l'article 220 n'est à l'ordre du jour que par la personnalisation du bénéficiaire de nouvelles dispositions constitutionnelles. Cette personnalisation aura déjà eu lieu à trois reprises. Primo, l'article 72 de la Constitution a fixé l'âge d'éligibilité à 30 ans, alors qu'il a toujours été historiquement stipulé à 40 ans au moins, en vue de permettre à un seul candidat de concourir à l'élection présidentielle à l'âge de 35 ans. D'ailleurs, sur les 33 candidats présidents à l'élection de 2006, aucun autre n'était âgé de moins de 40 ans. Secundo, l'élection présidentielle à un seul tour a été mise en place pour permettre à un seul candidat de bénéficier du meilleur classement et de l'emporter sans affronter un second tour. Tertio, la révision de l'article 70 est envisagée pour le seul candidat qui cumulerait plus de deux mandats successifs.

Cette personnalisation des règles constitutionnelles détruit totalement tout fondement de la notion d'Etat de droit, à savoir la nécessité de se soumettre aux règles juridiques établies. En effet, à quoi servent les règles si on ne les applique pas? Pourquoi doivent-elles être modifiées la première fois qu'elles trouvent une application? Cette personnalisation appelle trois considérations.

En premier lieu, le compte de deux mandats présidentiels successifs ne correspond pas aux faits. En 2016, le Président Joseph Kabila aura été investi quatre fois: en 2001, en 2003, en 2006 et en 2011. Le compte de seulement deux mandats est la conséquence juridique de la Constitution de 2006 qui a remis les compteurs à zéro. C'est ce que suggère la distinction

69 Alinéa 1.

70 C'est nous qui le soulignons. 
entre modification constitutionnelle et changement de constitution. Il suffirait d'habiller les choses de manière à créer une 'quatrième république' et le compteur serait remis à zéro. En deuxième lieu, les faits indiquent que le chef d'Etat actuel a accédé à la fonction suprême par filiation. Cette unique justification à sa désignation en 2001 place de facto la présidence congolaise dans un contexte monarchique où le pouvoir est passé de père en fils et aura totalisé près de vingt ans en 2016. Dès lors, la vraie question à poser clairement au souverain primaire, dans un référendum, serait qu'il se prononce sur l'instauration d'une monarchie de facto en RDC. En troisième lieu, la personnalisation des dispositions constitutionnelles, par deux fois, en 2006 et 2011, traduit l'adhésion des promoteurs d'une nouvelle révision au principe d'une 'monarchisation' de la présidence de la république ${ }^{71}$.

Il est difficile d'expliquer techniquement comment l'article 70 peut subir un amendement constitutionnel à l'occasion de sa toute première application. C'est un peu comme si les termes de la Constitution n'auraient servi à rien ; ce qui expliquerait qu'on se trouve en présence d'une "fraude à la Constitution" 72 pour permettre d'atteindre et de modifier les dispositions de l'article 70 ci-dessus.

A cet égard, deux opinions doctrinales ont été émises lors de débats juridiques de 2013 par deux professeurs de droit.

Une tendance a été lancée par le professeur Evariste Boshab. Celui-ci souligne que l'article 220 comporte plusieurs incohérences et qu'il n'est pas politiquement indispensable, parce qu'il n'ajoute rien quant à la détermination des citoyens à respecter et faire respecter l'application de la Constitution. ${ }^{73}$ Entre autres incohérences, il invoque la discrimination à l'égard du Chef de l'Etat, dans la mesure où les autres fonctions électives ne sont pas soumises à la même limitation du nombre et de la durée des mandats politiques : députés, sénateurs, gouverneurs de provinces, etc.. ${ }^{74} \mathrm{Il}$ y trouve aussi ce qu'il qualifie de "confiscation de la souveraineté [du peuple] par les élites ${ }^{175}$ parce qu'il prive le peuple, pourtant dans une démocratie, de voter pour qui il veut. ${ }^{76}$ Par conséquent, dit-il, il convient d'expurger cet article de la Constitution, qu'il considère comme ayant été imposé sous le dictat de la communauté internationale. ${ }^{77} \mathrm{Il}$ est soutenu en cela, par principe, par le professeur Jean-Louis Esambo, pour qui « l'immutabilité des dispositions constitutionnelles n'est pas d'applica-

71 Voir A. MBATA B. MANGU, « Monarchies présidentielles et révisions constitutionnelles: le syndrome du troisième mandat ou d'une présidence à vie dans les Etats membres de l'Union africaine ", Revue africaine de la démocratie et de la gouvernance, vol.I, n¹, 2014, pp.47-66.

72 Voir A. KAMUKUNI MUKINAY, Contribution à l'étude de la fraude en droit constitutionnel congolais, Louvain-la-Neuve, L'Harmattan-Academia, 2011.

73 E. BOSHAB, Entre la révision de la constitution et l'inanition de la nation, op.cit., p.332.

74 Idem, pp.347-348.

75 Id., p. 351.

76 Ibid.

77 Id., pp.331-332. 
tion absolue $»{ }^{78}$ Elles peuvent être modifiées, à condition que la révision « se fasse dans le strict but d'assurer l'intérêt général et respecte la procédure prévue par la Constitution ». ${ }^{79}$

Cette question peut être éclairée par des expériences africaines. En effet, la Constitution de l'Afrique du Sud avait interdit sa modification pendant les dix premières années de son application. La minorité blanche craignait la tyrannie constitutionnelle et législative de l'ANC. Passé ce délai, le parti de la majorité noire avait toujours le quorum requis pour procéder à des amendements, mais l'ANC n'en abusa pas et la Constitution n'a pas été modifiée. On n'en parle même pas. En Afrique du Sud, on a opté pour la stabilité des institutions plutôt que d'installer une suprématie politique à la faveur du vote d'une majorité parlementaire mécanique.

Deux pays africains ont recouru à la technique de la double révision constitutionnelle. Lorsque la constitution interdit une modification, on amende d'abord l'interdiction et dans un deuxième temps, on procède à la modification visée. Cette technique a permis de modifier la Constitution du Togo, en février 2005, pour permettre la succession monarchique au pouvoir du fils Eyadema à son feu père, Gnassingbé Eyadema. ${ }^{80}$ La méthode tourna mal au Niger lorsque le président Mamadou Tandja réussit à changer la Constitution pour s'octroyer la possibilité de décrocher un troisième mandat : il fut renversé par le coup d'Etat du 18 février 2010. En outre, le passage en force du président Blaise Compaoré, voulant modifier l'article 37 de la Constitution du Burkina Faso du 02 juin 1991 lui interdisant de briguer un troisième mandat consécutif, bien que non-verrouillé, a été déjoué par la révolte populaire de fin octobre 2014, qui l'a contraint à abandonner le pouvoir plutôt que prévu et à fuir précitamment vers la Côte d'Ivoire.

S'agissant de la RDC, les partisans du déverrouillage constitutionnel ne parviennent pas à expliquer par quel mécanisme licite on modifierait les articles 70 et 220 de la Constitution du 18 février 2008. On semble soutenir qu'un tel amendement, puisqu'il est interdit au constituant dérivé, doit être l'œuvre du seul constituant originaire. ${ }^{81}$ Pourtant, on oublie que, techniquement, celui-ci, n'ayant pas pour mission de modifier la Constitution, n'est pas du tout concerné par les procédures de révision constitutionnelle de l'article 218. Il ne faut pas que le recours au référendum soit mal compris à ce niveau, car le peuple intervient ici à un autre titre que celui du constituant originaire, dans le cadre et en respect de la Constitution. Il est ici constituant dérivé.

On peut, dès lors, comprendre la virulence très prononcée de l'autre tendance doctrinale, portée par le professeur André Mbata. Ce dernier est un positiviste qui ne veut pas

78 J.-L. ESAMBO KANGASHE, Le droit constitutionnel, Louvain-la-Neuve, Academia-L'Harmattan, 2013, p.103; La Constitution du 18 février 2006 à l'épreuve du constitutionnalisme. Contraintes pratiques et perspectives, Louvain-la-Neuve, Editions Academia Bruylant, 2010, p.97.

79 Ibid.

80 Voir R. ADJOVI, « Togo, un changement anticonstitutionnel savant et un nouveau test pour l'Union africaine ", Actualité et droit international, revue d'analyse juridique de l'actualité internationale, février 2005, pp.1-7 (disponible sur www.ridi.org/adi, consulté le 23 septembre 2013).

81 J.-L. ESAMBO KANGASHE, Le droit constitutionnel, op.cit., p.203. 
compliquer l'interprétation du texte constitutionnel, là où l'article 220 est clair et sans équivoque. Dans sa conférence du 26 juin 2013, à l'Université de Kinshasa, il contre-attaque et rejette la confusion qu'on veut entretenir entre constituant originaire et constituant dérivé. Il conclut à l'impossibilité de la révision de l'article 220 de la Constitution et met en cause la responsabilité des intellectuels congolais dans la consolidation de l'autoritarisme dans l'Etat. $^{82}$

Cette conférence du professeur Mbata a suscité deux actions: une pétition initiée par lui contre une nouvelle révision de la Constitution et une lettre ouverte du 03 septembre 2013, adressée au président Joseph Kabila. Il est appuyé en cela, dans de nombreuses prises de position au sein de la société civile congolaise, notamment l'Eglise catholique ${ }^{83}$ et la diaspora congolaise établie en Afrique du Sud. ${ }^{84}$ Les Nations unies, l'Union européenne, les Etats-Unis d'Amérique et d'autres partenaires extérieurs de la RDC ont aussi manifesté leur désapprobation. Peut-être la combinaison de ces initiatives pourrait-elle déjouer cette idée d'un nouvel amendement de la Constitution. Sous d'autres cieux, la justice, et en particulier la Cour constitutionnelle, serait la dernière véritable soupape de sécurité contre l'instrumentalisation du pouvoir constituant dérivé à des fins personnelles, alors qu'en RDC, l'exécutif a réussi, jusque-là, à mater le pouvoir judiciaire et à anéantir, par là même, toute son indépendance.

\section{L'anéantissement de l'indépendance du pouvoir judiciaire}

L'une des plus importantes innovations de la Constitution du 18 février 2006 est la proclamation explicite de l'indépendance du pouvoir judiciaire vis-à-vis du législatif et de l'exécutif. ${ }^{85}$ Ceci rappelle la théorie de séparation des pouvoirs telle qu'envisagée par Montesquieu $^{86}$ et tous les autres constitutionnalistes de l'époque contemporaine. Pour le constituant originaire, cette indépendance ne se limitait pas aux cours et tribunaux, car l'ancien article 149 de la Constitution incluait dans la composition du pouvoir judiciaire les parquets

82 A. MBATA B. MANGU, « Mandats présidentiels et révisions constitutionnelles en Afrique : la République démocratique du Congo dans la perspective de l'échéance 2016 », conférence de l'Institut pour la démocratie, la gouvernance, la paix et le développement en Afrique (IGDPA), Faculté de Droit, Université de Kinshasa, 26 juin 2013 (disponible sur http://pambazuka.org/fr/category/fe atures/88302, consulté le 23 septembre 2013).

83 CENCO, Mémorandum du comité permanent de la conférence épiscopale nationale du Congo au Président de la République sur l'état actuel de la nation, Kinshasa, 22 février 2013, point 18.

84 Voir Déclaration du 10 août 2013 de la diaspora congolaise établie en Afrique du Sud contre la révision de l'article 220 de la Constitution. Voir également la Déclaration du 25 août 2013 de la diaspora congolaise en Afrique du Sud concernant le dialogue national en préparation au pays, point 2.

85 Article 149, alinéa 1. Sur cette problématique, lire C. NGOMA KHUABI, « Analyse comparative de l'indépendance du pouvoir judiciaire en République démocratique du Congo et en République du Congo », Librairie africaine d'études juridiques, vol.6, août 2011, pp.51-92.

86 Voir C. MONTESQUIEU, Esprit des lois, tome 1, Paris, éd. Garnier Frères, 1973. 
qui y sont rattachés. ${ }^{87} \mathrm{Sa}$ gestion a été dévolue au Conseil supérieur de la magistrature (CSM), composé uniquement de magistrats ${ }^{88}$ et et doté formellement d'un budget propre, dont le premier président de la Cour de cassation est l'ordonnateur principal. ${ }^{89}$

Cependant, force est de constater le refus délibéré d'appliquer ce dispositif constitutionnel. Les entraves à l'exercice du pouvoir judiciaire sont devenues multiples et sophistiquées. Le président Joseph Kabila l'a lui-même reconnu en 2008 quand il affirme :

Très souvent, alors que les magistrats posent des actes conformément à leur devoir, et avant même qu'ils aient cloturé l'instruction préparatoire, des coups de fil pleuvent de partout pour paralyser l'action publique. Cela est inadmissible, au risque d'accréditer l'idée d'une République d'intouchables. ${ }^{90}$

En outre, on note des intimidations contre les magistrats à travers des révocations intempestives et mises à la retraite anticipée par le Chef de l'Etat, parfois sans passer par le CSM, ou par des mutations politiquement téléguidées par l'exécutif. En 2011, des nominations de hauts magistrats à la CSJ étaient intervenues en pleine campagne électorale pour l'élection présidentielle et ils avaient prêté serment devant un candidat dont ils devaient valider l'élection. ${ }^{91}$ A cette politisation à outrance de la gestion de la carrière des magistrats, ${ }^{92}$ il faut ajouter l'absence de budget propre pour le CSM, la modicité des crédits qui lui sont alloués par le pouvoir politique et le niveau assez bas des traitements du personnel judiciaire. Celui-ci est condamné à vivre, sinon de la charité des justiciables, du moins des primes qui leur sont, par mansuétude, attribuées par le pouvoir politique. ${ }^{93}$ Cette asphyxie financière $d u$ pouvoir judiciaire compte parmi les stratégies politiques qui font perdurer en RDC la situation dichotomique de l'existence du droit, symbolisé par un nombre impressionnant des lois adoptées par le Parlement, avec en même temps l'absence d'une réelle justice et de garantie effective de l'Etat de droit pour tous.

Cela n'étant pas suffisant pour maintenir la « dictature du politique sur le judiciaire $»,{ }^{94}$ il fut même envisagé, pour la première fois, à travers la proposition de révision constitutionnelle du député Tshibangu Kalala de 2007, de faire présider le CSM, non plus par le Président de la Cour constitutionnelle, mais par le Président de la République. Pareille ré-

\section{Alinéa 2.}

88 Article 152.

89 Article 149 in fine.

90 J. KABILA KABANGE, Discours sur l'état de la nation, Kinshasa, 13 décembre 2008.

91 M. YABILI, op.cit., p.295.

92 KIFWABALA TEKILAZAYA, DEFI FATAKI WA LUHINDI et M. WETSH'OKONDA KOSO, République démocratique du Congo : la justice et l'Etat de droit. Un Etat de droit en pointillé. Essai d'évaluation des efforts en vue de l'instauration d'un Etat de droit et perspectives, Johannesburg, Open Society Foundations, juillet 2013, p.73.

93 BALINGENE KAHOMBO, « La Cour suprême de Justice, Cour constitutionnelle transitoire ", http://www.la-constitution-en-afrique.org/, 2 février 2011.

94 Ibid. 
forme allait faire dépendre le pouvoir judiciaire de l'exécutif. En plus, la proposition était anticonstitutionnelle, puisqu'allant à l'encontre de l'article 220 de la Constitution qui fait figurer, on l'a vu, l'indépendance judiciaire sur la liste des matières qui ne peuvent faire l'objet d'aucune révision constitutionnelle.

Finalement, l'appareil judiciaire s'est complètement effondré avec l'amendement constitutionnel du 20 janvier 2011. Celui-ci a modifié l'article 149 en lui enlevant son alinéa 2 pour retirer les parquets de la composition du pouvoir judiciaire et restreindre celle-ci aux seuls cours et tribunaux. Cet amendement a permis de modifier l'organisation judiciaire et de stipuler que le Procureur général près la Cour de cassation obéit à certaines injonctions du Ministre de la justice. ${ }^{95}$

Le professeur Auguste Mampuya a regretté ce recul sur la voie de l'Etat de droit et la violation manifeste de l'article 220 de la Constitution en ces termes :

L'alinéa 2 de cet article [149] était ainsi une forte avancée de l'état de droit, en incluant les parquets dans le pouvoir judiciaire et en les faisant bénéficier de l'indépendance de leur corps. On pouvait alors chez nous résoudre une question que même les vieilles démocraties débattent aujourd'hui : celui d'un ministère public indépendant; nous étions en avance. Quand les auteurs de la proposition disent qu'il convient de réaffirmer "à cet effet la règle classique selon laquelle le parquet exerce son ministère sous l'autorité du ministre de la justice" "dont il est le bras séculier », ils oublient que l'option prise par le constituant de 2006 n'était pas une erreur ou un oubli mais le choix délibéré d'abandonner cette soi-disant «règle classique » en faveur de la conception moderne, que l'on trouve dans les pays anglo-saxons et que la France que nous aimons bien copier quand il s'agit de mauvais choix, est en train de mettre en place, sous le coup de condamnations répétées des instances internationales refusant de considérer ses procureurs comme " autorités judiciaires 》 et les considérant comme des organes non indépendants. ${ }^{96}$

Finalement, le constituant dérivé n'a pas poussé sa logique jusqu'au bout. Alors qu'il a retiré les parquets et, par voie de conséquence, tous les Officiers du ministère public (OMP), $\mathrm{du}$ pouvoir judiciaire, il s'est abstenu de définir leur nouveau statut, si tant est qu'ils doivent demeurer sous la dépendance de l'exécutif. Gardent-ils encore leur statut de magistrat, à côté des juges, tout en étant des agents gouvernementaux à la solde de l'exécutif? En réalité, cet amendement aurait dû s'accompagner de la modification de l'article 151 de la Constitution pour enlever tous les OMP de la composition du CSM. Celui-ci devrait désormais être l'apanage de seuls juges. Comme au Rwanda, où les parquets dépendent également de l'exécutif, il existe, pour eux, le Conseil supérieur du parquet, ${ }^{97}$ distinct du Conseil

95 Articles 72 et 98 de la loi organique du 11 avril 2013 portant organisation, fonctionnement et compétences des juridictions de l'ordre judiciaire.

96 A. MAMPUYA KANUNK'A-TSHIABO, « Au feu! L'article 220 violé », op.cit.

97 Article 165 de la Constitution du Rwanda du 4 juin 2003. 
supérieur de la magistrature. ${ }^{98}$ Il ne s'agit pas pour autant d'un modèle idéal d'organisation judiciaire à pouvoir copier, parce que, vues les fonctions du parquet, on arrive à conclure que, dans ce système, l'exécutif devient capable de dire qui dans l'Etat doit ou non faire l'objet de poursuites judiciaires. Ceci est évidemment une grosse erreur et une violation flagrante du principe juridique séculier de la séparation des pouvoirs. Ainsi, il convient de rétablir la formule de l'ancien article 149 de la Constitution et établir, en même temps, de nouveaux mécanismes permettant à la société de se prémunir contre le détournement persistant du pouvoir constituant dérivé.

\section{$V$. Les techniques constitutionnelles de prévention contre l'éventuel détournement persistant du pouvoir constituant dérivé}

Il convient de renforcer le dispositif constitutionnel pour que les méfaits des révisions constitutionnelles intempestives ne conduisent pas de l'institutionnalisation à la personnalisation encore durable du pouvoir politique, à la dictature, à la méconnaissance de l'Etat de droit et à la mort de la démocratie. ${ }^{99}$ Les mécanismes initiaux prévus par le constituant originaire ne sont plus suffisants, à savoir : l'article 220 de la Constitution qui peut, à nouveau, être violé à tout moment ; l'interdiction par l'article 219 de toute révision constitutionnelle en période exceptionnelle d'état de guerre, d'urgence ou de siège, ou pendant l'intérim à la présidence de la République ou lorsque l'Assemblée nationale et le Sénat se trouvent empêchés de se réunir librement.

Quelques nouvelles révisions constitutionnelles s'avèrent nécessaires. Premièrement, on peut renforcer la composition du pouvoir constituant dérivé par l'association des assemblées provinciales aux mécanismes de révision constitutionnelle. Cette proposition est inspirée de la Constitution de Luluabourg du $1^{\text {er }}$ août 1964 qui avait mis en place un fédéralisme à tendance unitaire. ${ }^{100}$ Evidemment, l'Etat actuel en détient déjà quelques caractéristiques, s'il n'est pas aussi fédéral ${ }^{101}$ ou quasi-fédéral, ${ }^{102}$ à défaut d'être considéré sous le

98 Idem, articles 157 et 158 .

99 Il faut, cependant, préciser que seul le droit ne pourrait pas suffire pour garantir la stabilité constitutionnelle, voire institutionnelle et politique, en RDC. Il faut aussi et surtout des hommes d'Etat respectueux de textes, qui ont déjà procédé à la « réforme des esprits ». Autant dire qu'il s'agit, avant tout, d'une question d'homme et de culture politique.

100 BALINGENE KAHOMBO, « L'expérience congolaise de l'Etat fédéral : la Constitution de Luluabourg revisitée », op.cit.

101 NYEMBO-Ya-LUMBU (O.), La Constitution de la Troisième République est fédérale. Regard critique sur la "décentralisation", Kinshasa, Editions Universitaires Africaines, 2009, p.7.

102 A. MBATA B. MANGU, "Perspectives du constitutionnalisme et de la démocratie en République Démocratique du Congo sous l'empire de la Constitution du 18 février 2006 », in BULABULA (S.) (dir.), Pour l'épanouissement de la pensée juridique congolaise. Liber Amicorum Marcel Antoine Lihau, Bruylant-PUK, Bruxelles-Kinshasa, 2006, p.212. 
prisme du régionalisme constitutionnel au niveau des provinces, ${ }^{103}$ coexistant avec la décentralisation et la déconcentration administrative au niveau des entités territoriales de base. ${ }^{104}$ Les provinces ayant leurs propres compétences, il est illusoire d'espérer qu'elles soient toujours respectées par le pouvoir central. Ce dernier renferme, à lui seul, une triple qualité : acteur de la décentralisation (exécutif et parlement), législateur national (parlement) et constituant dérivé (congrès). Il est dès lors compétent à la fois pour modifier la Constitution, édicter des normes légales nouvelles et concourir à leur application. Cette confusion des pouvoirs est malvenue, car il est largement admis que celui qui a le pouvoir, surtout tous les pouvoirs, a toujours tendance à en abuser. ${ }^{105}$ En introduisant les assemblées provinciales dans les procédures de révision constitutionnelle, elles pourraient mieux protéger les compétences des provinces et rendre plus difficiles, en d'autres matières, les tentatives d'instrumentalisation du constituant dérivé. Mais, il reste à trouver la formulation qui convient pour l'article 218 de la Constitution à modifier à cet effet. Pour autant, il ne s'agirait pas d'une innovation en soi en droit congolais, puisque la Loi fondamentale du 19 mai 1960 relative aux structures du Congo et la Constitution de Luluabourg du 1er août 1964 avaient aussi prévu des mécanismes juridiques similaires. ${ }^{106}$ Ces deux textes constitutionels pourraient ainsi servir de modèle d'inspiration.

En second lieu, on peut instaurer le système du contrôle obligatoire de la constitutionnalité des lois de révision constitutionnelle. En l'état actuel du droit congolais, un tel contrôle par le juge constitutionnel nécessite une interprétation savante de la Constitution. ${ }^{107}$

103 F. VUNDUAWE te PEMAKO, « Réflexion sur le régionalisme politique ou la nouvelle décentralisation territoriale dans la Constitution du 18 février 2006 : condition du développement des bases de l'Etat », Revue de la Faculté de Droit, n5, Droit et développement, actes des journées scientifiques organisées par la Faculté de Droit les 27 et 28 avril 2007, Editions de l'Université protestante au Congo, 2007, pp.181-219. A. KAMUKUNY MUKINAY et J. CIHUNDA HENGELELA, «Régionalisation, décentralisation et naissance effective des vingt-cinq nouvelles provinces en RD Congo : défis et perspectives de prévention des conflits ", Congo-Afrique, n ${ }^{4} 34$, avril 2009, pp.295-310.

104 Il s'agit des entités territoriales décentralisées (ETD) et des entités territoriales déconcentrées (ETDC). Les premières sont régies par la loi ${ }^{\circ} 08 / 016$ du 7 octobre 2008 portant composition, organisation et fonctionnement des entités territoriales décentralisées et leurs rapports avec l'Etat et les provinces. Les ETD sont : la ville, la commune, le secteur et la chefferie. En revanche, les secondes sont organisées par loi organique $\mathrm{n}^{\circ} 10 / 011$ du 18 mai 2010 portant fixation des subdivisions territoriales à l'intérieur des provinces. Les ETDC sont : le territoire, le quartier, le groupement et le village.

105 C. MONTESQUIEU, op.cit., cité par A. KAMUKUNY MUKINAY, Droit constitutionnel congolais, Kinshasa, Editions universitaires africaines, 2011, p.344.

106 Il s'agit de l'article 100 pour la Loi fondamentale et de l'article 176 pour la Constitution de Luluabourg.

107 BALINGENE KAHOMBO, «L'originalité de la Cour constitutionnelle congolaise: son organisation et sa compétence », op.cit., pp.14-17. 
En effet, on y distingue deux mécanismes techniquement différents de recours en appréciation de la constitutionnalité : le contrôle de constitutionnalité (articles 124, 139 et 160, alinéa 1, 2 et 3 ) et le recours en inconstitutionnalité (article 162, alinéa 2). Le premier a pour objet de faire déclarer, par voie d'action, un texte non encore promulgué ou qui n'est pas encore en vigueur conforme ou non à la Constitution. Il s'agit d'un contrôle préventif, c'est-à-dire a priori. Par contre, le second mécanisme, qui vise les textes législatifs ou réglementaires déjà mis en application, s'exerce nécessairement a posteriori, par voie d'action ou d'exception. Etant donné qu'une loi de révision constitutionnelle n'est pas matériellement et formellement un acte législatif, l'appréciation de sa constitutionnalité ne peut paset ne doit pas- être fondée sur l'article 162, alinéa 2, de la Constitution. La seule ouverture possible doit être recherchée dans le cadre du mécanisme du contrôle de constitutionnalité des lois. Comme celui-ci est nécessairement et absolument un contrôle préventif, le contrôle de constitutionnalité d'une loi de révision constitutionnelle devrait aussi s'exercer a priori.

Par ailleurs, la Constitution distingue entre le contrôle de constitutionnalité des lois organiques ${ }^{108}$ et celui des lois, ${ }^{109}$ sans aucune autre précision. Partant, elle ne semble pas du tout exclure du champ d'application du mécanisme de contrôle de constitutionnalité les lois de révision constitutionnelle, qui peuvent être rangées dans la catégorie des lois autres que celles organiques. Mais, pour éviter l'interprétation de la Constitution en fonction de la nature des conjonctures politiques, entraînant de facto une fluctuation et une instabilité des données jurisprudentielles, il est nécessaire de modifier son article 160 pour attribuer expréssement à la Cour constitutionnelle la compétence du contrôle obligatoire de constitutionnalité de toute loi de révision constitutionnelle.

Ainsi, comme le souligne Marcel Yabili ${ }^{110}$, le contrôle de constitutionnalité avant promulgation de toute loi, y compris celle de révision constitutionnelle, impliquerait, logiquement, la vérification de sa compatibilité avec la Constitution par application des principes de l'État de droit et de l'économie générale du dispositif constitutionnel. En effet, dans le principe, la Cour constitutionnelle ne remettrait pas en cause les normes constitutionnelles nouvelles, adoptées en remplacement des anciennes par le constituant dérivé. Mais, la révision constitutionnelle pourrait être irrégulière par irrespect d'autres normes constitutionnelles soit par violation des procédures, soit de règles substantielles non-modifiées et qui seraient contredites par la révision. ${ }^{11}$

En attendant, pareil recours non-obligatoire en vérification de la constitutionnalité d'une révision constitutionnelle peut effectivement être exercé. C'est l'apanage de l'opposition parlementaire, surtout parce qu'il est difficile de concevoir que la majorité qui a voté la

108 Article 124 et 160 , alinéa 2.

109 Article 139 et 160, alinéa 3.

110 M. YABILI, op.cit., pp.73-74.

111 Idem. 
loi en demande encore la censure auprès du juge constitutionnel. ${ }^{112}$ En pratique, il faut que cette opposition, bien que noyautée par une majorité mécanique, soit en mesure de réunir le dixième des députés ou des sénateurs nécessaires pour saisir la Cour constitutionnelle. L'important, bien entendu, est d'agir dans les délais avec des arguments bien structurés, tout en espérant que le juge constitutionnel, ayant le courage d'affirmer sa compétence en la matière, y prêtera son oreille attentive. Ce qui est loin d'être le cas jusque-là.

\section{Conclusion}

La démocratie et l'Etat de droit en RDC courent le risque de s'effondrer. Il se dessine autour du Chef de l'Etat une forte prégnance de confusion et de personnalisation du pouvoir d'Etat. En dépit du fait qu'il jouit déjà d'une ubiquité politique qui retentit sur la vie de toutes les institutions du pays, qui suivent ainsi la volonté d'un seul homme, voici qu'il est fait recours aux procédures de révision constitutionnelle dans l'unique but de les détourner à des fins purement personnelles, pour satisfaire les intérêts et les ambitions politiques d'un seul individu.

La révision du 20 janvier 2011 a présidentialisé la composition du constituant dérivé et celui-ci a tendance à accorder au Chef de l'Etat toujours plus de prérogatives constitutionnelles : dissolution des assemblées provinciales, révocation de gouverneurs de provinces, convocation du référendum constitutionnel, etc. L'appareil judiciaire tout entier, qui aurait pu être le protecteur de l'Etat de droit menacé, est lui-même asphyxié et paralysé par la politique. Le constituant dérivé a déjà par ailleurs réussi à amputer le corps judiciaire d'un de ses membres les plus importants que sont les parquets pour les rendre dépendants de l'exécutif national, commandé par le Chef de l'Etat. Tout puissant, et à l'abri du contrôle, il serait cependant risqué qu'il prétende confisquer le pouvoir politique en empêchant toute alternance démocratique et pacifique au fauteuil présidentiel. Les signes prémonitoires de ce pari risqué se sont déjà annoncés avec la modification du mode d“élection du Président de la République, peu avant le scrutin de novembre 2011. L'éventuelle modification de la durée et du nombre des mandats présidentiels, dont les débats ont commencé en 2013, pourrait constituer le point culminant d'une stratégie visant à lui garantir quasiment une présidence à vie, qui aura déjà duré près de 16 ans d'ici fin 2016. Dans un tel climat juridique, toute entreprise visant à amender telle ou telle autre disposition du texte constitutionnel devient l'objet de controverses et de souspicions politiques, à l'instar du projet gouvernemental du 9 juin 2014 suggérant l'élection des députés provinciaux au suffrage universel indirect par les élus locaux.

Face à cette situation, les possibilités de contre-stratégies sont bien minces. Faut-il croire en l'efficacité de la pression sociale qui parviendrait à imposer, faute de culture et de volonté politiques en la matière, le respect des textes, sans lequel il ne peut exister ni de

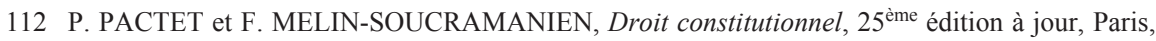
Sirey, 2006, p.76. 
démocratie ni d'Etat de droit digne de ce nom ? En outre, sur le plan juridique et constitutionnel, il est important d'instaurer des mécanismes de prévention d'un détournement persistant du pouvoir constituant dérivé à des fins purement personnelles. Ainsi, on pourrait, à travers la révision de l'article 218 de la Constitution, procéder à l'élargissement de la composition du pouvoir constituant dérivé aux assemblées provinciales. Celles-ci auraient, dès lors, la possibilité d'empêcher à l'échelon central, qui bénéficie actuellement d'une confusion des pouvoirs en matière des procédures de révision constitutionnelle, d'attenter, comme il veut, aux compétences des provinces ou à d'autres normes fondamentales qu'on peut considérer, à juste titre, comme constituant cette sorte de pacte constitutionnel national entre les différents échelons d'exercice des pouvoirs d'Etat (national, provincial et local) et les citoyens. Une autre solution, et pas la moindre, pourrait être la consécration du contrôle obligatoire de constitutionnalité de toute loi de révision constitutionnelle. Il faudrait donc modifier l'article 160 de la Constitution. 